\title{
Membrane targeting activates Leucine-rich repeat kinase 2 with differential effects on downstream Rab activation
}

Jillian H. Kluss ${ }^{1,2}$, Alexandra Beilina ${ }^{1}$, Patrick A. Lewis ${ }^{2,3,4}$, Mark R. Cookson ${ }^{1 *}$ and Luis Bonet-Ponce $^{1 *}$

\section{AFFILIATIONS}

${ }^{1}$ Cell Biology and Gene Expression Section, National Institute on Aging, National Institutes of Health, Bethesda, Maryland, USA.

${ }^{2}$ School of Pharmacy, University of Reading, Whiteknights, Reading, UK

${ }^{3}$ Royal Veterinary College, Royal College Street, London, UK.

${ }^{4}$ UCL Queen Square Institute of Neurology, Queen Square, London, UK.

${ }^{*}$ Correspondence: Luis Bonet-Ponce, Ph.D., Cell Biology and Gene Expression Section, Laboratory of Neurogenetics, National Institute on Aging, NIH, 35 Convent Drive, Room 1A-108, Bethesda, MD, 20892-3707, USA. E-mail: luis.bonet-ponce@nih.gov, ORCID: 0000-0001-9276-059X

${ }^{*}$ Correspondence: Mark R. Cookson, Ph.D., Cell Biology and Gene Expression Section, Laboratory of Neurogenetics, National Institute on Aging, NIH, 35 Convent Drive, Room 1A-116, Bethesda, MD, 20892-3707, USA. Phone: 301-451-3870 E-mail:

cookson@mail.nih.gov, ORCID: 0000-0002-1058-3831 


\section{ABSTRACT}

Genetic variation at the Leucine-rich repeat kinase 2 (LRRK2) locus contributes to risk of familial and sporadic Parkinson's disease. Recent data have shown a robust association between localization to various membranes of the endolysosomal system and LRRK2 activation. However, the mechanism(s) underlying LRRK2 activation at endolysosomal membranes are still poorly understood. Here we artificially direct LRRK2 to six different membranes within the endolysosomal system. We demonstrate that LRRK2 is activated and able to phosphorylate three of its Rab substrates (Rab10, Rab12 and Rab29) at each compartment. However, we report differing localization of pRab10 and pRab12 at the lysosomal and Golgi membranes. Specifically, we found that pRab10 colocalizes with a sub-population of perinuclear LRRK2-positive Golgi/lysosomal compartments whereas pRab12 localized to all LRRK2-positive Golgi/lysosomal membranes across the cell. When organelle positioning is manipulated by sequestering lysosomes to the perinuclear area, pRab10 colocalization with LRRK2 significantly increases. We also show recruitment of JIP4, a pRab10 effector that we have recently linked to LYTL, after trapping LRRK2 at various membranes. Taken together, we demonstrate that the association of LRRK2 to membranous compartments is sufficient for its activation and Rab phosphorylation independent of membrane identity. Our system also identifies a potential mechanism underlying the distinct relationships between LRRK2 and its substrates Rab10 and Rab12. 


\section{INTRODUCTION}

Mutations in the LRRK2 gene can cause familial Parkinson's Disease (PD), and non-coding variants in the promoter of the same gene act as risk factors for sporadic PD. LRRK2 encodes Leucine-repeat rich kinase 2 (LRRK2), a large protein where two enzymatic activities are encoded in a single molecule surrounded by protein-protein interaction domains. Known LRRK2 pathogenic mutations are located in the ROC-COR GTPase and kinase domains, and produce a toxic hyperactive protein (Greggio et al, 2006; West et al, 2005), leading to the proposal that kinase inhibitors may be useful to treat PD. Therefore, identifying the mechanism(s) by which LRRK2 becomes activated is of crucial interest to understand both disease risk and potential therapeutics.

Using a combination of cells expressing mutant LRRK2 and tool kinase inhibitors allowed Steger et al to identify a subset of Rab GTPases as direct LRRK2 substrates (Steger et al, 2016). LRRK2 phosphorylates Serine/Threonine residues in a conserved region of the switch II domain, leading to differential interactions with effector proteins, thus linking LRRK2 to vesicle mediated transport (Bonet-Ponce \& Cookson, 2019). Through these protein-protein interactions, LRRK2 has been shown to play a role at endomembranes including early endosomes, recycling endosomes, late endosomes, and lysosomes (Roosen \& Cookson, 2016; Hur et al, 2019; Yun et al, 2015).

Rab29, which is normally resident at the Golgi, is a LRRK2 substrate and has been shown in cells to promote the recruitment of LRRK2 to the trans-Golgi network (TGN) (MacLeod et al, 2013; Liu et al, 2018; Beilina et al, 2020, 2014). Rab29 engineering to contain a mitochondrial-targeting sequence is sufficient to bring LRRK2 to the mitochondrial membrane, suggesting Rab29 can control membrane recruitment 
of LRRK2 (Gomez et al, 2019). However, whether membrane specificity plays a role in LRRK2 activation and whether Rabs are required for LRRK2 activity remain unexplored.

In this study, we use the FK506 binding protein (FKBP) and the FKBPrapamycin-binding (FRB) domain (FKBP-FRB) (Robinson et al, 2010) to target LRRK2 to six different endomembranes and describe its activation pattern by measuring autophosphorylation at pS1292 LRRK2 and the phosphorylation of three endogenous Rab substrates pT73 Rab10, pS106 Rab12 and pT71 Rab29. Surprisingly, we observed that once directed to distinct membrane environments along the endolysosomal pathway, LRRK2 is activated and can phosphorylate all three Rab proteins, demonstrating that LRRK2 is able to recruit Rab substrates to each destination independent of membrane identity. pRab10 staining showed selective colocalization with LRRK2 at perinuclear lysosomal and Golgi membranes, whereas pRab12 was present at LRRK2-positive membranes regardless of its cellular location. Further manipulation of lysosomal positioning to the perinuclear area with the minus enddirected motor kinesin KIFC1 greatly enhanced the colocalization of pRab10 with LRRK2-positive lysosomes. We also confirmed recruitment of JIP4 to these membranes, an early step in LYsosomal Tubulation/sorting driven by LRRK2 (LYTL), and this could be blocked by treatment with a LRRK2 kinase inhibitor, MLi-2. We additionally demonstrate that mutant forms of LRRK2 show higher basal levels of activity and that while pS1292 LRRK2 and pRab10 further increase with membrane association, pRab12 does not. These results indicate that LRRK2 can be activated by recruitment to multiple endolysosomal membranes, but that there are divergent consequences for downstream effectors depending on localization. 


\section{RESULTS}

\section{LRRK2 can be activated at multiple endolysosomal membranes}

To study the activation of LRRK2, we took advantage of the FKBP-FRB system to trap LRRK2 to various membranes along the endolysosomal pathway. FKBP and FRB are both rapamycin-binding domains, originally from FKBP12, a propyl isomerase, and from mTOR, respectively. Thus, in the presence of rapamycin, these domains can form a heterodimer, and rapidly and irreversibly direct a target protein to the target membrane (Robinson et al, 2010) (Fig. 1A). We therefore tagged LRRK2 with the FKBP sequence (3xFLAG-FKBP-LRRK2 or mScarlet-FKBP-LRRK2), and target membrane markers with the FRB sequence. Since LRRK2 has been proposed to play a role at various membranous compartments within the endosomal system, we used FRBexpressed plasmids tagged with markers for plasma membrane (PM: PM(GAP43 ${ }_{P M-}$ targeting sequence)-FRB-CFP), early endosomes (EE: iRFP-FRB-RAB5), recycling endosomes (RE: EHD1-FRB-GFP), late endosomes (LE: iRFP-FRB-RAB7), lysosomes (LYS: LAMP1-FRB-CFP) and the Golgi network (Gol: FRB-CFP-Giantin) in order to interrogate LRRK2 activation across different membrane landscapes (Fig. 1B).

When exogenously expressed, LRRK2 presents a diffuse cytosolic distribution in most cell types (Beilina et al, 2014; Stafa et al, 2012; Mamais et al, 2014) . Cotransfection of 3xFlag-FKBP-LRRK2 with an FRB-tagged plasmid containing LAMP1, Giantin, or EHD1 in HEK293FT cells resulted in diffuse LRRK2 localization. However, we were able to demonstrate colocalization of LRRK2 to the membranes of lysosomes, Golgi, and recycling endosomes, respectively, after treatment with rapamycin (Fig. 1C; 
Movie S1; Movie S2; Fig. S1B-C). Similar experiments were performed where LRRK2 was redistributed to membranes of early endosomes, late endosomes or the plasma membrane (Fig. S1A). These results demonstrate that we can control localization of LRRK2 to different endolysosomal membranes using the FKBP-FRB system.

We next asked whether LRRK2 was activated when directed to each membrane. A significant increase in S1292 LRRK2 phosphorylation was observed via Western blot compared to control cells that were not treated with rapamycin irrespective of membrane targeted (Fig. 2A-B,F,J; Fig. S2A-B,F,J). Incubation with MLi-2, a potent LRRK2 kinase inhibitor, decreases S1292 phosphorylation signal, confirming that the observed signal is kinase-dependent. Neither exposure to rapamycin nor solvent (ethanol) alone resulted in LRRK2 activation, demonstrating that the increase in pS1292 is a direct result of membrane binding (Fig. S3). We next wanted to characterize downstream activation patterns of known LRRK2 substrates, hypothesizing that pRab profiles would be membrane-dependent given that each membrane has a unique Rab signature. Surprisingly, we found that membrane-bound LRRK2 phosphorylates Rab10, Rab12, and Rab29 at each respective phospho-site T73, S106, and T71, irrespective of the particular membrane in which LRRK2 was trapped (Fig. 2, Fig. S2). Additionally, MLi-2 treatment prevented Rab phosphorylation thus confirming its dependence on LRRK2 activity. Collectively, these results indicate that directing LRRK2 to any given membrane results in activation and phosphorylation of multiple Rabs, irrespective of membrane identity. 


\section{A lysosome directed LRRK2 chimera reproduces activation patterns seen in the FKBP-FRB system}

Our data indicate that neither the membrane identity, nor binding to a resident Rab GTPase are required for LRRK2 activation at a membrane. Most of our traps are proteins that have no previous link to LRRK2 i.e. LAMP1, Giantin, EHD1, and GAP43Pmtargeting sequence although Rab5 has been nominated as a LRRK2 substrate (Steger et al, 2016). Thus, we hypothesize that LRRK2 does not require a specific protein interaction to undergo activation at a membrane. In order to further evaluate this hypothesis, we developed a second method of targeting LRRK2 to a membrane by tagging LRRK2 to the $\mathrm{N}$ terminal domain of LAMTOR1 (aa1-39). LAMTOR1 is a member of the Ragulator complex and controls activation of mTORC1 in the lysosomal membrane and the first 39 amino acids of LAMTOR1 are sufficient to target any protein to the lysosomal membrane (Amick et al, 2018; Menon et al, 2014; Amick et al, 2020). We confirmed Lyso-LRRK2 (LAMTOR1(1-39)-3xFLAG-LRRK2) localization to the lysosomal membrane by colocalization with endogenous LAMP1 (Fig. 3A). The autophosphorylation of LRRK2 in cells transfected with Lyso-LRRK2 were comparable to that of the Lys-trap with rapamycin treatment and higher than Lys-trap alone, suggesting that the presence of LRRK2 at a membrane is sufficient for its activation

(Fig. 3B, C). Phosphorylation of pT73 Rab10 and pS106 Rab12 were also significantly higher in cells expressing the Lyso-LRRK2 plasmid compared to Lys-trap LRRK2 alone (untreated) (Fig. 3B, D, E). Of particular note, the Lyso-LRRK2 chimera strongly increased pT71-Rab29 (Fig. 3F). 


\section{LRRK2 does not require Rab29 for activation at a membrane}

It is noteworthy that Rab29, previously described as being restricted to the TGN, was phosphorylated when LRRK2 was directed to non-Golgi membranes. Rab29 has been proposed to be a master regulator of LRRK2 activity by promoting its recruitment to the TGN (Purlyte et al, 2019; Gomez et al, 2019). As we see activation at membranes that would be predicted not to have Rab29, but do in fact recover pRab29 at those membranes, it is possible that Rab29 controls LRRK2 activity more generally than just at the TGN. To test this hypothesis, we knocked down endogenous Rab29 prior to cotransfection of LRRK2 and the Gol-trap containing Giantin. This resulted in a significant increase in S1292, Rab10, and Rab12 phosphorylation when treated with rapamycin but there was no significant effect of Rab29 depletion compared to non-targeting siRNA (Fig. 4). This data demonstrates that LRRK2 activation is independent of Rab29.

\section{Phosphorylation of Rab10 and Rab12 differ in localization patterns at the} lysosomal and Golgi membranes

In subsequent experiments, we chose to focus on trapping LRRK2 to the lysosome or Golgi based on the literature reporting that LRRK2 influences cell biology via Rab substrates at these membranes (Beilina et al, 2014; Purlyte et al, 2019; Herbst et al, 2020; Eguchi et al, 2018; Bonet-Ponce et al, 2020). Available phospho-antibodies for pT73 Rab10 and pS106 Rab12 were validated in immunostaining experiments to be able to visualize the localization of these LRRK2 substrates (in our hands pT71 Rab29 did not give a reliable staining). Both T73 Rab10 and S106 Rab12 showed increased 
staining and colocalization with LRRK2 at the lysosomal and Golgi membranes after cotransfecting LRRK2 and the Lys-trap or the Gol-trap respectively and treatment with rapamycin. After additional treatment with $\mathrm{MLi}-2$, this phosphorylation was lost (Fig. 5A, B). To quantify the ratio of colocalization of LRRK2 to pRab10 and pRab12, we used Airyscan super-resolution confocal microscopy. Surprisingly, we found differing localization patterns between pRab12 and pRab10. pRab12 was highly colocalized to LRRK2-positive lysosomes or Golgi membrane throughout the cells, whereas pRab10 was found to colocalize only in perinuclear subsets of lysosomes and Golgi ( $2.5-3$ fold lower colocalization to LRRK2 compared to pRab12) (Fig. 5C - E). Furthermore, this striking difference was specific only to the lysosomal and Golgi membranes, as early and recycling endosomes showed similar high colocalization to LRRK2 between pRab10 and pRab12 (Fig. 5E, Fig. S4A - D). This suggests there may be differences in membrane composition at the lysosome and Golgi that affect LRRK2-dependent phosphorylation of Rab10 to a greater extent than Rab12.

\section{Lysosomal sequestration to the perinuclear region favors $p$ Rab10 localization at LRRK2-positive lysosomes}

These data show that pT73 Rab10 exhibits a specifically perinuclear localization with LRRK2 at the lysosomal membrane, whereas pS106 Rab12 colocalizes with almost all LRRK2-positive lysosomes. Lysosomes are highly regulated organelles and can be moved in both anterograde and retrograde directions along microtubules depending on the cell's need for active lysosomes (Cabukusta \& Neefjes, 2018; Ballabio 
\& Bonifacino, 2020). In several studies characterizing lysosomes, it is thought that perinuclear lysosomes are most active, as the lumenal $\mathrm{pH}$ is highly acidic (Johnson et al, 2016) ( 4.0-4.8) and contain enzymes (Cheng et al, 2018; Yap et al, 2018), creating the most efficient conditions for degradation. Based on these data, we speculated that lysosomal positioning may be a factor in the distribution of $\mathrm{pRab} 10$. To evaluate this hypothesis, we employed the reversible association with motor proteins (RAMP) system in order to manipulate lysosomal positioning (Guardia et al, 2019). Briefly, we introduced a truncated version of the minus end-directed motor protein KIFC1 construct fused with a LAMP1 construct affixed with a streptavidin binding site. As a result, all lysosomes in cells transfected with the LAMP1-SBP-GFP and Strep-KIFC1*-HA constructs are sequestered to the perinuclear region (Fig. 6A-B).

Using this method, we co-transfected these constructs together with our LysoLRRK2 chimera and stained for endogenous pT73 Rab10 and pS106 Rab12. Surprisingly, we observed a significant increase in the colocalization between LRRK2 and pRab10 with the addition of KIFC1 compared to conditions transfected with LysoLRRK2 and LAMP1 alone, whereas pS106 Rab12 remained highly colocalized with LRRK2 in either condition (Fig. 6C-E). It is worth noting that the signal intensity of pRab10 did not change between conditions but pRab12 signal decreased slightly with the addition of KIFC1 compared to the control condition (Fig. 6F-G). This suggests that lysosomes located in the perinuclear region give rise to conditions optimal for LRRK2dependent Rab10 phosphorylation. 


\section{JIP4 is recruited to perinuclear LRRK2-positive membranes}

We and others have confirmed JIP4, a motor adaptor protein, as a downstream interactor of pRab10 (Waschbusch et al, 2020) that can be recruited to membrane damaged lysosomes (Bonet-Ponce et al, 2020). To determine whether the FKBP-FRB trap system could also induce the membrane recruitment of a pRab effector, we stained for endogenous JIP4 after co-transfection of LRRK2 and the Lys-trap or Gol-trap. Under untreated conditions, JIP4 shows diffuse expression throughout the cytoplasm. After treatment with rapamycin, JIP4 is found to colocalize with LRRK2 at the lysosomal and Golgi membranes, which is LRRK2 kinase-dependent as it can be reversed by MLi-2 treatment (Fig. 7A, B). These JIP4 puncta are only found at the most perinuclear membranes, similar to pT73 Rab10 (Fig. 7C, D). Similar distributions of pRab10 and JIP4 were noted using the EE-trap (Fig. S5A, B). We also evaluated JIP4 localization using the Lyso-LRRK2 chimera plasmid and found that JIP4 also behaves in the same fashion as with the Lys-trap system (Fig. S5C). Additionally, when observed after lysosomal positioning manipulation, we found that JIP4 colocalized more with LRRK2 when more LRRK2 was within the perinuclear area after co-transfection with the LAMP1-SBP-GFP and Strep-KIFC1*-HA constructs (Fig. 7F).

We have previously shown that the recruitment of JIP4 by LRRK2 to damaged lysosomes triggers a tubulation and sorting process called LYTL (Bonet-Ponce et al, 2020). Here we explored whether the presence of LRRK2 to the lysosomal membrane was sufficient to trigger JIP4 tubulation. We used the Lyso-LRRK2 plasmid to make sure we were not imaging spontaneous events that occurred before rapamycin addition, and were able to detect sporadic JIP4 tubules in cells expressing Lyso-LRRK2 (Fig. 
7E). These results collectively indicate that recruitment of LRRK2, particularly to lysosomal membranes, is sufficient to induce the initiating events of LYTL.

\section{Pathogenic mutations of LRRK2 show divergent patterns of activation between $p$ Rab10 and pRab12 at membranes}

We next wanted to determine whether LRRK2 pathogenic mutations would alter Rab phosphorylation at the Golgi and lysosomal membranes in our system. We created FKBP-tagged LRRK2 with R1441C, Y1669C (gain-of-function mutations present in the ROC-COR domain), G2019S (gain-of-function mutation present in the kinase domain) and K1906M (kinase dead) mutations. As expected, in the absence of rapamycin, R1441C, Y1669C and G2019S increased the autophosphorylation site pS1292 LRRK2, with the G2019S mutation showing the highest increase compared to the GTPase domain mutations in both traps (Fig. 8A, B, E, F). The artificial kinase dead control K1906M showed no S1292 phosphorylation. Interestingly, although the G2019S mutation has the most influence on $\mathrm{S} 1292$ signal, the $\mathrm{Y} 1669 \mathrm{C}$ mutation has a stronger influence on LRRK2 pRab substrates (Steger et al, 2016; Ito et al, 2016) which we confirmed here (Fig. 8A, C-E, G). In the presence of rapamycin, a significant increase in phosphorylation at T73 Rab10 was observed in the context of every LRRK2 mutant variant at both Golgi and lysosomal membranes (Fig. 8A, C, E, G). Interestingly, this was not the case with pS106 Rab12 in which mutant LRRK2 resulted in non-significant differences between their untreated and rapamycin treated counterparts (Fig. 8A, D, E, H). These results show that mutations in LRRK2 are hyperactive, as expected, but that 
their membrane association can provoke additional activity depending on which Rab substrate is being measured.

\section{DISCUSSION}

Understanding the patterns of LRRK2 kinase activity is of crucial interest for PD pathogenesis and future therapeutics targeted at LRRK2. Recent work from our lab and others have shown that under specific stimuli, LRRK2 can be targeted to different membranous compartments where it can phosphorylate and recruit several Rab substrates. For example, LRRK2 has been robustly shown to translocate to the TGN, the phagosomal membrane and the membrane of damaged lysosomes depending on the stimulus used (Beilina et al, 2014; Herbst et al, 2020; Lee et al, 2020; Eguchi et al, 2018; Bonet-Ponce et al, 2020; Purlyte et al, 2019). However, the dynamics underlying LRRK2 membrane presence, activation and Rab phosphorylation remain unclear and, specifically, whether membrane association is necessary or sufficient for LRRK2 activation.

Here we take advantage of the FKBP-FRB system to target LRRK2 to six different endomembranes, enabling us to study the subsequent events that follow LRRK2 membrane recruitment to the plasma membrane, early endosomes, recycling endosomes, late endosomes, lysosomes and the TGN. We found that LRRK2 undergoes activation, as measured by autophosphorylation at S1292, irrespective of the membranous compartment. Additionally, we noted a similar increase in phosphorylation 
of T73 Rab10, S106 Rab12 and T71 Rab29 at all membranes studied, suggesting that firstly, membrane identity is not important for LRRK2 activationand, secondly pRabs do not seem to exhibit membrane preferences, but rather can be recruited by LRRK2 to each membrane the kinase is present at. This data is largely consistent with recent observations using Rab29 to drive LRRK2 to membranes other than the TGN (Gomez et al, 2019) that was suggested to occur due to GDI1/2 proteins being unable to capture phosphorylated Rabs away from membranes.

We also find that LRRK2 activation at membranes is Rab29-independent. Although co-overexpression of LRRK2 and Rab29 promotes the recruitment of LRRK2 to the TGN (Beilina et al, 2014, 2020; Liu et al, 2018), leading to the idea that Rab29 is a regulator of LRRK2 activation and localization (Purlyte et al, 2019), recent data using Rab29 knockout models did not identify a corresponding decrease in Lrrk2 activity in the confirmed absence of Rab29 (Kalogeropulou et al, 2020). Here, we find that LRRK2 is able to undergo activation in each membrane to which it is directed without addition of Rab29 and its depletion did not alter LRRK2 kinase activation once LRRK2 was trapped at the Golgi. Furthermore, Rab29 was phosphorylated by LRRK2 at all of the membranes we have analyzed, similar to Rab10 and Rab12.

The tagging of a lysosomal targeting sequence (LAMTOR1(aa1-39)) to LRRK2 was sufficient to trigger LRRK2 activation, which strongly indicates that the required signal to activate LRRK2 is its presence at a membranous compartment rather than binding to Rab proteins per se. Nonetheless, because there are multiple, phosphorylated Rabs localised to each membrane, it remains possible that multiple Rab proteins may be able to redundantly substitute for Rab29 in various membrane 
compartments. More work is therefore required to understand the signals that mediate LRRK2 recruitment to endomembranes under physiological conditions. We speculate that Rab29 overexpression may result in a form of membrane damage at the TGN leading to recruitment that is functionally similar to the events we have recently described at the lysosome with membrane damaging agents (Eguchi et al, 2018; Herbst et al, 2020; Bonet-Ponce et al, 2020).

Additionally, we characterized pRab localization patterns after LRRK2 was trapped to the lysosomal and Golgi membranes. We observed a striking difference in colocalization pattern with LRRK2, with pT73 Rab10 showing strict perinuclear localization in a subset of lysosomes and Golgi membranes whereas pS106 Rab12 was found in almost all LRRK2-positive compartments throughout the cell. In a recent study from our lab, we propose pRab12 as a better biomarker for LRRK2 kinase activity than pRab10 in vivo, demonstrating wide variability in the response of pRab10 to MLi-2 compared to pRab12 (Kluss et al). The current data suggests that perhaps the pool of Rab12 is either more readily available to LRRK2 once at a membrane and/or that there is a mechanism specific to Rab10 at select membrane subsets that makes it amenable to LRRK2 manipulation. Interestingly, JIP4 followed the same perinuclear localization as pT73 Rab10 in these membranes, confirming the link between pRab10 and JIP4, as we have shown in the membrane of dysfunctional lysosomes (Bonet-Ponce et al, 2020). The recruitment of LRRK2, pRab10 and JIP4 to ruptured lysosomes results in the formation of JIP4-positive tubules that we were able to partially recreate by simply translocating LRRK2 to lysosomes. It is likely that other signals, besides LRRK2 presence, are needed for the full recruitment of JIP4 and pRab10 to LRRK2-positive 
membranes. One such characteristic may be lysosomal positioning, as forced placement of lysosomes to the perinuclear area via the minus-end motor kinesin KIFC1 was sufficient enough to significantly increase colocalization between LRRK2 and pRab10. How this positioning promotes LRRK2-dependent pRab10 recruitment warrents further investigation. For example, the mechanisms whereby pRab10 is preferentially localised to a perinuclear subset of LRRK2-positive lysosomes or Golgi, but is able to accompany LRRK2 to early and recycling endosomes wherever their position within the cell remain unclear.

In the context of LRRK2 pathogenic mutations, we observed differing patterns of influence on pRab10 and pRab12. Phosphorylation of Rab10 was significantly increased after addition of rapamycin as expected, however, phosphorylation of Rab12 was not further increased by rapamycin treatment within the LRRK2 mutant backgrounds. This effect only occurred in the context of mutant LRRK2, as WT LRRK2 showed additional phosphorylation with rapamycin treatment. This result, along with our observation that pRab12 strongly colocalizes with almost all LRRK2-positive lysosomes in the Lyso-LRRK2 context, suggests that Rab12 is readily available for phosphorylation by membrane-bound LRRK2. Studies have shown that mutant LRRK2 is present at membranes more frequently than wildtype LRRK2. Thus, it is plausible that mutant LRRK2 alone is enough to phosphorylate Rab12 such that the addition of a membraneFRB plasmid to trap LRRK2 to the membrane does not further increase Rab12 phosphorylation.

The discrepancy in phosphorylation patterns of Rab10 and Rab12 in the context of LRRK2 GTPase and kinase mutants raises a question as to whether differing Rabs 
play distinct roles that may lead to PD pathogenesis. Rab GTPases are highly conserved proteins, and many studies have tried to elucidate their specific functional roles within the cell (Roosen \& Cookson, 2016; Zhen \& Stenmark, 2015). Further experimental models and tools will need to be explored in order to better define the roles of Rab10 and Rab12 in PD pathology.

Overall, we have shown that membrane identity is not required for LRRK2 activation, nor is the binding to Rab29. Our results suggest that the presence of LRRK2 to a membrane is sufficient to trigger its activation, Rab recruitment and its subsequent phosphorylation. These events were also sufficient for downstream recruitment of JIP4. By inference, this data suggests that processes including LYTL emerge from the association of LRRK2 with various endomembrane compartments. A limitation of the current study is that we have used artificial constructs, albeit utilising multiple different approaches, to drive LRRK2 to various endolysosomal membranes. Although our results provide new information on how membrane presence triggers LRRK2 activation, further research at physiologically relevant levels of expression is required to gain a deeper understanding of this crucial process.

Collectively, our data outline new aspects of the regulation of LRRK2 recruitment and activity at different membranous systems in cells. Further evaluation of this hypothesis, and the identification of physiological regulators of this process, may be instructive for better understanding of early events in PD pathogenesis.

\section{ACKNOWLEDGEMENTS}


This research was supported by the Intramural Research Program of the NIH, National Institute on Aging (MRC) and the University of Reading. We thank every member from the Cookson lab for critical feedback. We also thank Rosa Puertollano and Tsukasa Okiyoneda for kindly providing the CFP-FRB-LAMP1 and the EHD1-FRB-GFP plasmids, respectively. We thank Carlos M. Guardia and Juan S. Bonifacino for the gift of the strep-KIFC1*-HA and LAMP1-SBP-GFP constructs.

\section{AUTHOR CONTRIBUTIONS}

Conceptualization, L.B-P., M.R.C., J.H.K.; Methodology, L.B-P., J.H.K., A.B.; Formal

Analysis, J.H.K., L.B-P.; Investigation, J.H.K., L.B-P.; Writing, L.B-P., J.H.K., M.R.C.;

Supervision, L.B-P., M.R.C., P.A.L.; Funding, M.R.C., P.A.L.

\section{DECLARATION OF INTERESTS}

The authors have no competing interests related to this work. 


\section{MATERIAL AND METHODS}

\section{Cell culture}

HEK293FT cells were maintained in DMEM containing $4.5 \mathrm{~g} / \mathrm{l}$ glucose, $2 \mathrm{mM} \mathrm{I-}$ glutamine, $5 \%$ Pen/Strep and $10 \% \mathrm{FBS}$ at $37^{\circ} \mathrm{C}$ in $5 \% \mathrm{CO}_{2}$. HEK293FT cells were seeded on $12 \mathrm{~mm}$ coverslips pre-coated with matrigel.

\section{Reagents and treatments}

For all experiments, rapamycin (Cayman Chemicals, cat \#13346) was added at $200 \mathrm{nM}$ in ethanol for $15 \mathrm{~min}$ before fixing or lysing cells for downstream analyses. Addition of MLi-2 (Tocris, cat \#5756) was used at $1 \mu \mathrm{M}$ in DMSO, 90 min before rapamycin treatment. HaloTag-RAB10 transfected cells were incubated with the JF646 peptide (Promega, cat \#GA1120) at $200 \mathrm{nM}$ for $15 \mathrm{~min}$, cells were then washed $3 x$ and incubated with fresh media before treating with rapamycin. For the lysosomal positioning experiments, NeutrAvidin (ThermoFisher, cat \#31000) was added to the media at $0.5 \mathrm{mg} / \mathrm{ml}$ concentration periodically before fixing for biotin quenching, as the presence of biotin can disrupt the interaction between LAMP1 and KIFC1, making this interaction reversible.

\section{Cloning}

FKBP sequence was tagged to 3xFLAG-pDEST and mScarlet-pDEST vectors using IN-FUSION HD cloning technology (Clontech, Takara, cat \#638920). LRRK2-WT, LRRK2-R1441C, LRRK2-Y1669C, LRRK2-K1906M and LRRK2-G2019S, previously cloned into $\mathrm{pCR}^{\mathrm{TM}} 8 / \mathrm{GW} / \mathrm{TOPO}^{\mathrm{TM}}$ vector (ThermoFisher, cat \#250020), were transferred 
into the 3xFLAG-FKBP-pDEST and mScarlet-FKBP-pDEST plasmids using Gateway technology (ThermoFisher, cat \#11791043). LYSO-LRRK2 was created by adding the $\mathrm{N}$ terminal domain of the LAMTOR1 sequence (aa 1-39) into the 3xFLAG-LRRK2 plasmid using IN-FUSION (LAMTOR1(1-39)-3xFLAG-LRRK2). CFP-FRB-LAMP1 vector (Willett et al, 2017) was a gift from Rosa Puertollano (NIH). EHD1-FRB-GFP was kindly provided by Tsukasa Okiyoneda (Kwansei Gakuin University). PM-FRB-CFP (Addgene plasmid \#67517) (Varnai et al, 2006), iRFP-FRB-RAB7 (Addgene plasmid \#51613) and iRFP-FRB-RAB5 plasmids (Addgene plasmid \#51612) (Hammond et al, 2014) were gifts from Tamas Balla (NIH). FRB-ECFP-Giantin was a provided by Dorus Gadella (Addgene plasmid \#67903) (van Unen et al, 2015). The LAMP1-SBP-GFP and Strep$\mathrm{KIFC} 1^{*}$-HA plasmids were a generous gift from Juan S. Bonifacino (Guardia et al, 2019).

Transfection and siRNA knockdown

Transient transfections of HEK293FT cells were performed using Lipofectamine 2000 in Gibco's Opti-MEM (ThermoFisher, cat \#31985088). HEK293FT cells were transfected for $24 \mathrm{~h}$. For endogenous Rab29 knockdown experiments, cells were treated with either non-targeting control siRNA or human Rab29 siRNA together with Lipofectamine RNAiMAX (ThermoFisher, cat \#13778075) in Gibco's Opti-MEM and incubated for $24 \mathrm{~h}$ prior to starting the transfection protocol. For the lysosomal positioning experiments, transfection media was replaced with fresh media containing neutravidin at $0.5 \mathrm{mg} / \mathrm{mL}$ two hours after transfection for all conditions. 


\section{Antibodies}

The following primary antibodies were used: mouse anti-FLAG M2 (SigmaAldrich, cat \#F3165, 1:500 for ICC and 1:10,000 for WB), mouse anti-LAMP1 (DSHB, cat \#H4B3, 1:100 for ICC), rat anti-FLAG (Biolegend, cat \#637302, 1:200 for ICC), chicken anti-GFP (AvesLab, GFP-1020, 1:1,000 for ICC), mouse anti-GFP (Roche, cat \#11814460001, 1:10,000 for WB), rabbit anti-RAB10 (Abcam, cat \#ab237703, 1:2,000 for WB), rabbit anti-RAB10 (phospho-T73) (Abcam, cat \#ab23026, 1:200 for ICC and 1:2,000 for WB), rabbit anti-RAB12 (Proteintech, cat \#18843-1-AP, 1:1,000 for WB), rabbit anti-RAB12 (phospho-S106) (Abcam, cat \#ab256487, 1:100 for ICC and 1:2,000 for WB), rabbit anti-RAB29 (Abcam, cat \#ab256526, 1:2,000 for WB), rabbit anti-RAB29 (phospho-T71) (Abcam, cat \#ab241062, 1:2,000 for WB), rabbit anti-LRRK2 (Abcam, cat \#ab133474, 1:2,000 for WB), rabbit anti-LRRK2 (phospho S1292) (Abcam, cat \#ab203181, 1:2,000 for WB), rabbit anti-EEA1 (Cell Signaling Technology, cat \#3288, 1:100 for ICC), rabbit anti-cyclophilin B (Abcam, cat \#ab16045, 1:2,000 for WB), sheep anti-TGN46 (Biorad, cat \#AHP500GT, 1:500 for ICC), rabbit anti-Rab8a (Cell Signaling Technology, cat \#6975, 1:500 for ICC), rabbit anti-Lamtor4 (Cell Signaling Technology, cat\#12284, 1:200 for ICC), rabbit anti-JIP4 (Cell Signaling Technology, cat\#5519, 1:100 for ICC), chicken anti-HA (Millipore Sigma, cat \#AB3254, 1:250 for ICC) . For staining of the plasma membrane, Phalloidin was used at 1:20 concentration to visualize F-actin (ThermoFisher, cat \#A30107).

For ICC, unless otherwise stated, the secondary antibodies were purchased from ThermoFisher. The following secondary antibodies were used: donkey anti-mouse Alexa-Fluor 568 (cat \#A10037, 1:500), donkey anti-rabbit Alexa-Fluor 488 (cat \#A- 
21206, 1:500), donkey anti-mouse Alexa-Fluor 568 (cat \#A-21202, 1:500), donkey antirat Alexa-Fluor 488 (cat \#A-21208, 1:500), donkey anti-goat Alexa-Fluor 488 (cat \#A11055, 1:500), donkey anti-rabbit Alexa-Fluor 568 (cat \#A10042, 1:500), donkey antimouse Alexa-Fluor 647 (cat \#A-31571, 1:500), goat anti-rat Alexa-Fluor 647 (cat \#A21247, 1:250-1:500). Donkey anti-chicken Alexa-Fluor 405 (cat \#703-475-155, 1:300) was obtained from Jackson ImmunoResearch.

For WB, all secondary antibodies were used at 1:10,000 dilution: IRDyes 800CW Goat anti-Rabbit IgG (Licor, cat \#926-32211) and 680RD Goat anti-Mouse IgG (Licor, cat \#926-68070). All blots presented in each figure panel were derived from the same experiment and were processed in parallel.

\section{Confocal microscopy}

Confocal images were taken using a Zeiss LSM 880 microscope equipped with a 63X 1.4 NA objective. Super-resolution imaging was performed using the Airyscan mode. Raw data were processed using Airycan processing in 'auto strength' mode with Zen Black software version 2.3. Only low plasmid expression cells without obvious overexpression artifacts were imaged. For measuring colocalization, Fiji plugin JACoP was used in which Mander's correlation corrected for threshold was used to quantify LRRK2:pRab colocalization. Colocalized maps were made using the Colocalization Finder plugin in Fiji. For measuring signal intensity, integrated density of each cell was measured using Fiji without thresholding (ImageJ, NIH).

Immunostaining 
HEK293FT cells were fixed with 4\% PFA for 10 mins, permeabilized with PBS/ 0.1\% Triton for 10 mins and blocked with 5\% Donkey Serum (Sigma, cat \#D9663) for 1 h at RT. Primary antibodies were diluted in blocking buffer (1\% Donkey Serum) and incubated overnight at $4^{\circ} \mathrm{C}$. After three 5 min washes with PBS/ $0.1 \%$ Triton, secondary fluorescently labeled antibodies were diluted in blocking buffer (1\% Donkey Serum) and incubated for 1 hour at RT. Coverslips were washed two times with 1x PBS and an additional $2 x$ with $\mathrm{dH}_{2} \mathrm{O}$, and mounted with ProLong® Gold antifade reagent (ThermoFisher, cat \#P10144).

\section{SDS PAGE and Western Blotting}

Proteins were resolved on 4-20\% Criterion TGX precast gels (Biorad, cat \#5671095) and transferred to nitrocellulose membranes (Biorad, cat \#170415) by semidry trans-Blot Turbo transfer system (Biorad). The membranes were blocked with Odyssey Blocking Buffer (Licor, cat \#927-40000) and then incubated for $1 \mathrm{~h}$ at RT or overnight at $4^{\circ} \mathrm{C}$ with the indicated primary antibody. The membranes were washed in TBST $(3 \times 5 \mathrm{~min})$ followed by incubation for $1 \mathrm{~h}$ at RT with fluorescently conjugated secondary antibodies as stated above (Licor). The blots were washed in TBST ( $3 \times 5$ min) and scanned on an ODYSSEY ${ }^{\circledR}$ CLx (Licor). Quantitation of western blots was performed using Image Studio (Licor).

\section{Statistical analysis}

Analyses based on cell counts were performed by an investigator blinded to treatment/transfection status. Statistical analysis for experiments with two treatment 
groups used Unpaired Student's t-tests. For more than two groups, we used one-way ANOVA or two-way ANOVA where there were two factors in the model. Tukey's posthoc test was used to determine statistical significance for individual comparisons in those cases where the underlying ANOVA was statistically significant and where all groups' means were compared to every other mean. Unless otherwise stated, graphed data are presented as means \pm SD or SEM. Comparisons were considered statistically significant where $p<0.05 .{ }^{*}, p<0.05 ;{ }^{* *}, p<0.01 ;{ }^{* * *}, p<0.001 ;{ }^{* * * *}, p<0.0001$. 


\section{FIGURE LEGENDS}

Figure 1. The FRB-FKBP system can be used to trap LRRK2 to membranes. (A)

Schematic showing the mechanism behind FRB-FKBP binding. In the presence of rapamycin, the FKBP fragment forms a heterodimer with the FRB fragment via their rapamycin binding domains. Thus, co-transfection of FKBP-LRRK2 and FRB-membrane plasmids allows us to trap LRRK2 to membranes and investigate downstream signaling events. (B) Schematic of the plasmid components cloned. Fusion of 1) FRB fragments to known proteins of specific membrane organelles and 2) the FKBP fragment to LRRK2. Key: EE = Early endosomes; LE = Late endosomes, Gol = Golgi, Lys $=$ Lysosome, $\mathrm{PM}=$ Plasma membrane, $\mathrm{RE}=$ Recycling endosomes. $(\mathrm{C})$ Representative confocal images of HEK293FT cells co-transfected with 3xFlag-FKBP-LRRK2 (red) and CFP-FRB-Lamp1, GFP-FRB-Giantin, or GFP-FRB-EHD1 (green) show colocalization at the lysosomal, Golgi, and recycling endosomal membranes in the presence of rapamycin, respectively. Untreated groups show cytosolic localization of LRRK2. Lamtor4, TGN46, and Rab8a (grey) were used as endogenous markers for each respective membrane. Scale bar $=10 \mu \mathrm{m}$.

Figure 2. Membrane-bound LRRK2 activates its autophosphorylation and subsequent phosphorylation of Rab substrates. Co-transfection of 3xFlag-FKBPLRRK2 and CFP-FRB-Lamp1, GFP-FRB-Giantin, or GFP-FRB-EHD1 in HEK293FT cells. (A) Once localized to the lysosomal, Golgi, or recycling endosomal membrane, western blot analysis revealed activation of LRRK2 kinase via S1292 autophosphorylation as well as an increase in phosphorylation of T73 Rab10, S106 
Rab12, and T71 Rab29, which was ameliorated after MLi-2 treatment (B - M). (B - M:

one-way ANOVA with Tukey's post hoc, ${ }^{* * *} P<0.0001,{ }^{* * *} P<0.0009,{ }^{* *} P<0.005,{ }^{*} P$ $<0.0332 ; n=3$; SD bars shown; $(\mathrm{B}) F(2,6)=116.4$, (C) $F(2,6)=81.12$, (D) $F(2,6)=$ 376.3, (E) $F(2,6)=13.89),(F) F(2,6)=116.4,(\mathrm{G}) F(2,6)=81.12,(\mathrm{H}) F(2,6)=376.3$, (I) $F(2,6)=13.89),(\mathrm{J}) F(2,6)=104.6,(\mathrm{~K}) F(2,6)=110.5,(\mathrm{~L}) F(2,6)=48.55$, (M) $F(2$, $6)=15.71$. N. Cartoon depicting our model where LRRK2 undergoes autophosphorylation and is able to phosphorylate Rab10, Rab12 and Rab29 irrespective of membrane identity.

Figure 3. Lysosomal targeting of LRRK2 via chimera yields a similar activation pattern to the FRB-FKBP system. Activation patterns of LRRK2 using the 3xFlagFKBP-LRRK2/CFP-FRB-Lamp1 co-transfection (Lys-trap) treated with rapamycin and LRRK2 chimera-only transfection (Lyso-LRRK2) were compared. (A) Representative confocal microscopy images show colocalization of LRRK2 (red) to LAMP1 (green) in Lys-trap and Lyso-LRRK2 models. (B) Western blot analyses show an increase in S1292 LRRK2 phosphorylation and subsequent Rab substrates in both the Lys-trap model treated with rapamycin and the Lyso-LRRK2 model. (C - F) Statistical analyses show similar degrees of phosphorylation. C - F: one-way ANOVA with Tukey's post hoc, ${ }^{* * * *} P<0.0001,{ }^{* * *} P<0.0009,{ }^{* *} P<0.005,{ }^{*} P<0.0332 ; n=3$; SD bars shown; (C) $F(2,6)=87.10$, (D) $F(2,6)=65.83$, (E) $F(2,6)=16.40$, (F) $F(2,6)=39.04$. Scale bar= $10 \mu \mathrm{m}$. 
Figure 4. Knockdown of endogenous Rab29 does not affect LRRK2 activation at the lysosomal membrane. Following a $24 \mathrm{~h}$ incubation of siRNA, HEK293FT cells were transfected with 3xFlag-FKBP-LRRK2 and Golgi trap. No difference was observed in pS1292, pT73 Rab10, nor pS106 Rab12 signals in cells given Rab29 siRNA as compared to NTC. With the addition of rapamycin, significant increases in phosphorylation were observed in both Rab29 and NTC siRNA comparably (A - D). B D: two-way ANOVA with Tukey's multiple comparisons test, ${ }^{* * *} P<0.0001,{ }^{*} P<0.05 ; n$ $=3$; SD bars shown; $(B)$ treatment, $p<0.0001, F(1,8)=214.9$; siRNA, $p=0.1536, F(1$, $8)=2.485,(C)$ treatment, $p<0.0001, F(1,8)=267.3$; siRNA, $p=0.6008, F(1,8)=$ 0.2967, (D) treatment, $p<0.0019, F(1,8)=20.69$; siRNA, $p=0.1269, F(1,8)=2.901$.

Figure 5. pT73 Rab10 and pS106 Rab12 are phosphorylated by LRRK2 at membranous organelles. Utilizing the FRB-FKBP system, representative confocal microscopy images show colocalization of LRRK2 and its Rab substrates at membranes. (A) Using the Lys-trap (green), both pS106 Rab12 and pT73 Rab10 (red) colocalize with LRRK2 (grey) at the lysosomal membrane when treated with rapamycin and are dephosphorylated with MLi-2 treatment. These events are recapitulated in the Gol-trap (green) (B). Interestingly in both traps, pS106 Rab12 colocalizes perfectly with all LRRK2 staining whereas pT73 Rab10 shows a particularly perinuclear localization pattern (C - E) Colocalized pixels are shown for clarity of colocalization. E: Two-way ANOVA with Tukey's multiple comparisons test, $n=9-10$ cells; SEM bars shown; $\mathrm{pRab}$ comparison, $p<0.0001, F(1,53)=110.1$; Trap, $p<0.0001, F(1,53)=50.29$. Scale bar $=$ $10 \mu \mathrm{m}(\mathrm{A}, \mathrm{B}) ; 5 \mu \mathrm{m}(\mathrm{C}, \mathrm{D})$. 
Figure 6. LRRK2-positive lysosomes sequestered to the perinuclear area via KIFC1 is sufficient to increase LRRK2 colocalization with pRab10 and JIP4. Lyso-LRRK2 and LAMP1-SBP-GFP were co-transfected with or without Strep-KIFC1* HA and supplemented with neutrAvidin for $24 \mathrm{hrs}(\mathrm{A}, \mathrm{B})$. Colocalization of LRRK2 with pS106 Rab12 remained consistently high after moving all lysosomes to the perinuclear region $(C, E)$ whereas pT73 RAb10 colocalization with LRRK2 significantly increased after lysosomes were sequestered (D, E). Interestingly pRab10 intensity was preserved whereas pRab12 signal intensity was reduced (F-G). E: two-way ANOVA with Tukey's multiple comparisons test, $n=9-13$ cells; SD bars shown; KIFC1 presence, $p<0.0001$, $F(1,39)=24.32$; phospho-site, $p=0.0156, F(1,39)=6.400$. F-G: Unpaired t test (twotailed), $n=13-16$ cells; SD bars shown; $(\mathrm{F}) p=0.6336,(\mathrm{G}) p=0.0358$. Scale bar $=10$ $\mu \mathrm{m}(\mathrm{A}, \mathrm{B}) ; 5 \mu \mathrm{m}(\mathrm{C}, \mathrm{D})$.

Figure 7. Recruitment of JIP4 is observed after trapping LRRK2 to the lysosomal and Golgi membranes. To investigate whether any events downstream of Rab10 are observed in this trap system, we stained for JIP4 after trapping LRRK2 to the lysosomal (A) and Golgi (B) membranes using rapamycin. Interestingly, JIP4 shows a similar pattern to the pT73 Rab10 staining, in which JIP4 showed partial colocalization with LRRK2 in particularly perinuclear lysosomes and Golgi membranes (C, D, respectively.). (E) JIP4 was also found to have increased presence in LRRK2-positive lysosomes when lysosomes were clustered towards the perinuclear area using the KIFC1 construct. (F) Using Airyscan super-resolution confocal microscopy, we were 
able to detect sporadic JIP4 tubules (blue arrowheads) in cells expressing Lyso-LRRK2 (F). Scale bar= $10 \mu \mathrm{m}(\mathrm{A}, \mathrm{B}) ; 5 \mu \mathrm{m}(\mathrm{C}-\mathrm{F}) .(\mathrm{G})$. Cartoon depicting our model of membrane-bound LRRK2 phosphorylation of Rab substrates depending on their cellular localization.

Figure 8. LRRK2 and Rab phosphorylation patterns are affected by LRRK2 pathogenic mutations at the Golgi and lysosomal membranes. The Gol-trap and Lys-trap was co-transfected with either WT, R1441C, Y1669C, G2019S, or K1906M LRRK2(-FKBP-3xFlag or mScarlet) varieties. When treated with rapamycin, S1292 LRRK2 showed an increase in phosphorylation in all PD-linked mutations, with the largest increase observed in the G2019S mutation. No phosphorylation in the K1906M kinase dead variant $(A, B, E, F)$. The highest phosphorylation signals of pT73 Rab10 and pS106 Rab12 were observed with transfection of the Y1699C LRRK2 variant, regardless of treatment $(C-D, G-H)$. With respect to treated versus untreated conditions, T73 Rab10 shows a significant increase in phosphorylation within all mutant conditions, whereas pS106 Rab12 seems to show a ceiling effect with mutations regardless of rapamycin treatment. MLi-2 was used as a negative control for all phospho-proteins shown. B - D, F - H: two-way ANOVA with Tukey's multiple comparisons test, ${ }^{* * *} P<0.0001,{ }^{* * *} P<0.0009,{ }^{* *} P<0.005,{ }^{*} P<0.05 ; n=3$; SD bars shown; (B) treatment, $p<0.0001, F(2,30)=177.0$; genotype, $p<0.0001, F(4,30)=$ 121.5, $(C)$ treatment, $p<0.0001, F(2,30)=310.7$; genotype, $p<0.0001, F(4,30)=$ 156.0, (D) treatment, $p<0.0001, F(2,30)=210.1$; genotype, $p<0.0001, F(4,30)=$ 117.9, $(F)$ treatment, $p<0.0001, F(2,30)=208.2$; genotype, $p<0.0001, F(4,30)=$ 
130.8, $(\mathrm{G})$ treatment, $p<0.0001, F(2,30)=200.5$; genotype, $p<0.0001, F(4,30)=$ 95.63, $(\mathrm{H})$ treatment, $p<0.0001, F(2,30)=97.52$; genotype, $p<0.0001, F(4,30)=$ 45.79 .

\section{Supplementary Figure 1. EE, LE, PM, RE, and Lys FRB-plasmids trap LRRK2 to} membranes. (A) Representative confocal images of HEK293FT cells co-transfected with 3xFlag-FKBP-LRRK2 (red) and iRFP-FRB-Rab5, iRFP-FRB-Rab7 (grey), or CFPFRB-PMTS (green) show colocalization at the early endosome, late endosome, and plasma membranes in the presence of rapamycin, respectively. Untreated groups show cytosolic localization of LRRK2. EEA1, LAMP1 (green), and F-actin (grey) were used as endogenous markers for each respective membrane. Images taken from live cell imaging experiments visualize the rapid localization of LRRK2 (magenta) to the recycling endosomal $(B)$ and lysosomal $(C)$ membranes (green) after treatment with rapamycin around 144s and 215.6s, respectively. Scale bar= $10 \mu \mathrm{m}(\mathrm{A}) ; 5 \mu \mathrm{m}(\mathrm{B}, \mathrm{C})$.

\section{Supplementary Figure 2. Membrane-bound LRRK2 activates its} autophosphorylation and subsequent phosphorylation of Rab substrates. Cotransfection of 3xFlag-FKBP-LRRK2 and iRFP-FRB-Rab5, iRFP-FRB-Rab7, or CFPFRB-PMTS in HEK293FT cells. (A) Once localized to the early endosomal, late endosomal, or plasma membrane, western blot analysis revealed activation of LRRK2 kinase via S1292 autophosphorylation as well as an increase in phosphorylation of T73 Rab10, S106 Rab12, and T71 Rab29, which was ameliorated with MLi-2 treatment (B M). (B - M: one-way ANOVA with Tukey's post hoc, ${ }^{* * *} P<0.0001,{ }^{* * *} P<0.0009,{ }^{* *} P$ 
$<0.005,{ }^{*} P<0.0332 ; n=3$; SD bars shown; (B) $F(2,6)=69.67$, (C) $F(2,6)=36.89$, (D)

$F(2,6)=26.02,(\mathrm{E}) F(2,6)=3.874,(\mathrm{~F}) F(2,6)=22.40,(\mathrm{G}) F(2,6)=691.5$, (H) $F(2,6)=$

48.89, (I) $F(2,6)=45.89,(J) F(2,6)=119.8,(\mathrm{~K}) F(2,6)=154.4,(L) F(2,6)=277.7,(\mathrm{M})$

$F(2,6)=103.9$.

\section{Supplementary Figure 3. Rapamycin alone is not sufficient to bring LRRK2 to a} membrane without a trap plasmid co-transfection. For QC purposes, LRRK2 was transfected with and without the Golgi trap, and under treated and untreated conditions. When both LRRK2 and trap are co-expressing, only conditions of rapamycin increase S1292 and S106 Rab12 phosphorylation. Treatment with ethanol at 1:1,000, the rapamycin carrier solvent, did not affect phosphorylation of LRRK2 nor Rab12.

\section{Supplementary Figure 4. Additional colocalization of pT73 Rab10 and pS106}

Rab12 at the early and recycling endosomal membranes. Utilizing the FRB-FKBP system, representative confocal microscopy images show colocalization of LRRK2 and its Rab substrates at the early and recycling endosomal membranes. (A) Using the REtrap or EE-trap (green), both pS106 Rab12 and pT73 Rab10 (red) colocalize with LRRK2 (grey) at the lysosomal membrane when treated with rapamycin and as well as are dephosphorylated with $\mathrm{MLi}-2$ treatment $(\mathrm{A}, \mathrm{B})$. In both early and recycling endosome traps, pS106 Rab12 as well as pT73 Rab10 colocalize with all LRRK2 staining $(C, D)$. This is notably different in the lysosome and Golgi trap, where pT73 Rab10 was only colocalized to a subset of LRRK2 puncta, suggesting differing 
membrane-specific properties. Colocalized pixels are shown for clarity of colocalization.

Scale bar= $10 \mu \mathrm{m}(\mathrm{A}, \mathrm{B}) ; 5 \mu \mathrm{m}(\mathrm{C}-\mathrm{E})$.

\section{Supplementary Figure 5. Trapping of LRRK2 via FKBP-FRB or lyso-LRRK2 \\ chimera results in the recruitment of JIP4 at the lysosomal and early endosomal}

membranes. To look at JIP4 recruitment to other membranes, the EE-trap and FKBP-

LRRK2 were co-transfected in HEK293FT cells. With addition of rapamycin, JIP4

recruitment was seen to the early endosomal membrane, with MLi-2 treatment blocking this event (A). Airyscan imaging shows colocalization of LRRK2 and JIP4 (B). The use of the Lyso-LRRK2 chimera is sufficient to recruit JIP4 to the lysosomal membrane compared to WT-LRRK2 plasmid. With the addition of MLi-2 treatment, JIP4 recruitment is not visible $(C)$. Scale bar= $10 \mu \mathrm{m}(A, C) ; 5 \mu \mathrm{m}(B)$.

\section{Supplementary Movie 1. Live imaging of LRRK2 and RE-trap colocalizes with} addition of rapamycin. HEK293FT cells expressing mScarlet-FKBP-LRRK2, and RE trap (EHD1-FRB-GFP) were treated with rapamycin. Time lapse was taken using an inverted confocal microscope at 9 seconds/ frame (video is played at 6 frames/ second). At time $144 \mathrm{~s}$, the rapamycin was added to the cells in order to view the colocalization of cytosolic LRRK2 to the RE membrane in real time. Images were taken in a single plane.

\section{Supplementary Movie 2. Live imaging of LRRK2 and Lys-trap colocalizes with} addition of rapamycin. HEK293FT cells expressing mScarlet-FKBP-LRRK2, and Lys trap (LAMP1-FRB-CFP) were treated with rapamycin. Time lapse was taken using an 
bioRxiv preprint doi: https://doi.org/10.1101/2020 12.01.406223. this version posted December 2 2020. The copyright holder for this preprint (which was not certified by peer review) is the author/funder. This article is a US Government work. It is not subject to copyright under 17 USC 105 and is also made available for use under a CCO license.

inverted confocal microscope at 71.86 seconds/ frame (video is played at 6 frames/ second). At time $359.3 \mathrm{~s}$, the rapamycin was added to the cells in order to view the colocalization of cytosolic LRRK2 to the Lys membrane in real time. Images are maximum intensity projection from a z-stack. 


\section{REFERENCES}

Amick J, Tharkeshwar AK, Amaya C \& Ferguson SM (2018) WDR41 supports lysosomal response to changes in amino acid availability. Mol Biol Cell 29: 22132227

Amick J, Tharkeshwar AK, Talaia G \& Ferguson SM (2020) PQLC2 recruits the C9orf72 complex to lysosomes in response to cationic amino acid starvation. J Cell Biol 219

Ballabio A \& Bonifacino JS (2020) Lysosomes as dynamic regulators of cell and organismal homeostasis. Nat Rev Mol Cell Biol 21: 101-118

Beilina A, Bonet-Ponce L, Kumaran R, Kordich JJ, Ishida M, Mamais A, Kaganovich A, Saez-Atienzar S, Gershlick DC, Roosen DA, et al (2020) The Parkinson's Disease Protein LRRK2 Interacts with the GARP Complex to Promote Retrograde Transport to the trans-Golgi Network. Cell Rep 31: 107614

Beilina A, Rudenko IN, Kaganovich A, Civiero L, Chau H, Kalia SK, Kalia LV, Lobbestael E, Chia R, Ndukwe K, et al (2014) Unbiased screen for interactors of leucine-rich repeat kinase 2 supports a common pathway for sporadic and familial Parkinson disease. Proc Natl Acad Sci U S A 111: 2626-2631

Bonet-Ponce L, Beilina A, Williamson CD, Lindberg E, Kluss JH, Saez-Atienzar S, Landeck N, Kumaran R, Mamais A, Bleck CKE, et al (2020) LRRK2 mediates tubulation and vesicle sorting from lysosomes. Sci Adv 6

Bonet-Ponce L \& Cookson MR (2019) The role of Rab GTPases in the pathobiology of Parkinson' disease. Curr Opin Cell Biol 59: 73-80

Cabukusta B \& Neefjes J (2018) Mechanisms of lysosomal positioning and movement. Traffic 19: 761-769

Cheng X-T, Xie Y-X, Zhou B, Huang N, Farfel-Becker T \& Sheng Z-H (2018) Characterization of LAMP1-labeled nondegradative lysosomal and endocytic compartments in neurons. J Cell Biol 217: 3127-3139

Eguchi T, Kuwahara T, Sakurai M, Komori T, Fujimoto T, Ito G, Yoshimura S-I, Harada A, Fukuda M, Koike M, et al (2018) LRRK2 and its substrate Rab GTPases are sequentially targeted onto stressed lysosomes and maintain their homeostasis. Proc Natl Acad Sci U S A 115: E9115-E9124

Gomez RC, Wawro P, Lis P, Alessi DR \& Pfeffer SR (2019) Membrane association but not identity is required for LRRK2 activation and phosphorylation of Rab GTPases. J Cell Biol 218: 4157-4170 
Greggio E, Jain S, Kingsbury A, Bandopadhyay R, Lewis P, Kaganovich A, van der Brug MP, Beilina A, Blackinton J, Thomas KJ, et al (2006) Kinase activity is required for the toxic effects of mutant LRRK2/dardarin. Neurobiol Dis 23: 329-341

Guardia CM, De Pace R, Sen A, Saric A, Jarnik M, Kolin DA, Kunwar A \& Bonifacino JS (2019) Reversible association with motor proteins (RAMP): A streptavidin-based method to manipulate organelle positioning. PLoS Biol 17: e3000279

Hammond GRV, Machner MP \& Balla T (2014) A novel probe for phosphatidylinositol 4phosphate reveals multiple pools beyond the Golgi. J Cell Biol 205: 113-126

Herbst S, Campbell P, Harvey J, Bernard EM, Papayannopoulos V, Wood NW, Morris HR \& Gutierrez MG (2020) LRRK2 activation controls the repair of damaged endomembranes in macrophages. EMBO J: e104494

Hur E-M, Jang E-H, Jeong GR \& Lee BD (2019) LRRK2 and membrane trafficking: nexus of Parkinson's disease. BMB Rep 52: 533-539

Ito G, Katsemonova K, Tonelli F, Lis P, Baptista MAS, Shpiro N, Duddy G, Wilson S, Ho PW-L, Ho S-L, et al (2016) Phos-tag analysis of Rab10 phosphorylation by LRRK2: a powerful assay for assessing kinase function and inhibitors. Biochem $J$ 473: 2671-2685

Johnson DE, Ostrowski P, Jaumouillé V \& Grinstein S (2016) The position of lysosomes within the cell determines their luminal pH. J Cell Biol 212: 677-692

Kalogeropulou AF, Freemantle JB, Lis P, Vides EG, Polinski NK \& Alessi DR (2020) Endogenous Rab29 does not impact basal or stimulated LRRK2 pathway activity. Biochem J

Kluss JH, Mazza MC, Li Y, Manzoni C, Lewis PA, Cookson MR \& Mamais A Preclinical Modeling of Chronic Inhibition of the Parkinson's Disease Associated Kinase LRRK2 Reveals Altered Function of the Endolysosomal System in Vivo. doi:10.21203/rs.3.rs-78203/v1 [PREPRINT]

Lee H, Flynn R, Sharma I, Haberman E, Carling PJ, Nicholls FJ, Stegmann M, Vowles J, Haenseler W, Wade-Martins R, et al (2020) LRRK2 Is Recruited to Phagosomes and Co-recruits RAB8 and RAB10 in Human Pluripotent Stem Cell-Derived Macrophages. Stem Cell Reports 14: 940-955

Liu Z, Bryant N, Kumaran R, Beilina A, Abeliovich A, Cookson MR \& West AB (2018) LRRK2 phosphorylates membrane-bound Rabs and is activated by GTP-bound Rab7L1 to promote recruitment to the trans-Golgi network. Hum Mol Genet 27: 385-395

MacLeod DA, Rhinn H, Kuwahara T, Zolin A, Di Paolo G, McCabe BD, Marder KS, Honig LS, Clark LN, Small SA, et al (2013) RAB7L1 Interacts with LRRK2 to Modify Intraneuronal Protein Sorting and Parkinson's Disease Risk. Neuron 77: 994 
doi:10.1016/j.neuron.2013.02.021 [PREPRINT]

Mamais A, Chia R, Beilina A, Hauser DN, Hall C, Lewis PA, Cookson MR \& Bandopadhyay R (2014) Arsenite stress down-regulates phosphorylation and 14-33 binding of leucine-rich repeat kinase 2 (LRRK2), promoting self-association and cellular redistribution. J Biol Chem 289: 21386-21400

Menon S, Dibble CC, Talbott G, Hoxhaj G, Valvezan AJ, Takahashi H, Cantley LC \& Manning BD (2014) Spatial control of the TSC complex integrates insulin and nutrient regulation of mTORC1 at the lysosome. Cell 156: 771-785

Purlyte E, Dhekne HS, Sarhan AR, Gomez R, Lis P, Wightman M, Martinez TN, Tonelli F, Pfeffer SR \& Alessi DR (2019) Rab29 activation of the Parkinson's diseaseassociated LRRK2 kinase. EMBO J 38

Robinson MS, Sahlender DA \& Foster SD (2010) Rapid inactivation of proteins by rapamycin-induced rerouting to mitochondria. Dev Cell 18: 324-331

Roosen DA \& Cookson MR (2016) LRRK2 at the interface of autophagosomes, endosomes and lysosomes. Mol Neurodegener 11: 73

Stafa K, Trancikova A, Webber PJ, Glauser L, West AB \& Moore DJ (2012) GTPase activity and neuronal toxicity of Parkinson's disease-associated LRRK2 is regulated by ArfGAP1. PLoS Genet 8: e1002526

Steger M, Tonelli F, Ito G, Davies P, Trost M, Vetter M, Wachter S, Lorentzen E, Duddy G, Wilson S, et al (2016) Phosphoproteomics reveals that Parkinson's disease kinase LRRK2 regulates a subset of Rab GTPases. Elife 5

van Unen J, Reinhard NR, Yin T, Wu YI, Postma M, Gadella TWJ \& Goedhart J (2015) Plasma membrane restricted RhoGEF activity is sufficient for RhoA-mediated actin polymerization. Sci Rep 5: 14693

Varnai P, Thyagarajan B, Rohacs T \& Balla T (2006) Rapidly inducible changes in phosphatidylinositol 4,5-bisphosphate levels influence multiple regulatory functions of the lipid in intact living cells. J Cell Biol 175: 377-382

Waschbusch D, Purlyte E, Alessi DR \& Khan AR (2020) Crystal structure of phosphorylated Rab8a in complex with the Rab-binding domain of RILPL2. doi:10.2210/pdb6rir/pdb [PREPRINT]

West AB, Moore DJ, Biskup S, Bugayenko A, Smith WW, Ross CA, Dawson VL \& Dawson TM (2005) Parkinson's disease-associated mutations in leucine-rich repeat kinase 2 augment kinase activity. Proc Natl Acad Sci U S A 102: 16842-16847

Willett R, Martina JA, Zewe JP, Wills R, Hammond GRV \& Puertollano R (2017) TFEB regulates lysosomal positioning by modulating TMEM55B expression and JIP4 recruitment to lysosomes. Nat Commun 8: 1580 
Yap CC, Digilio L, McMahon LP, Garcia ADR \& Winckler B (2018) Degradation of dendritic cargos requires Rab7-dependent transport to somatic lysosomes. $J$ Cell Biol 217: 3141-3159

Yun HJ, Kim H, Ga I, Oh H, Ho DH, Kim J, Seo H, Son I \& Seol W (2015) An early endosome regulator, Rab5b, is an LRRK2 kinase substrate. J Biochem 157: 485495

Zhen Y \& Stenmark H (2015) Cellular functions of Rab GTPases at a glance. J Cell Sci 128: $3171-3176$ 

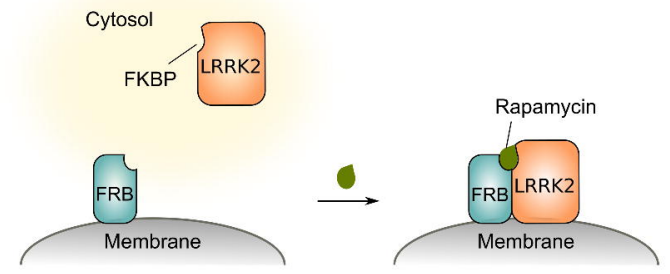

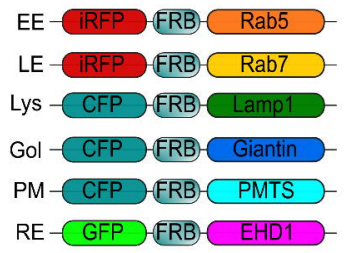

C

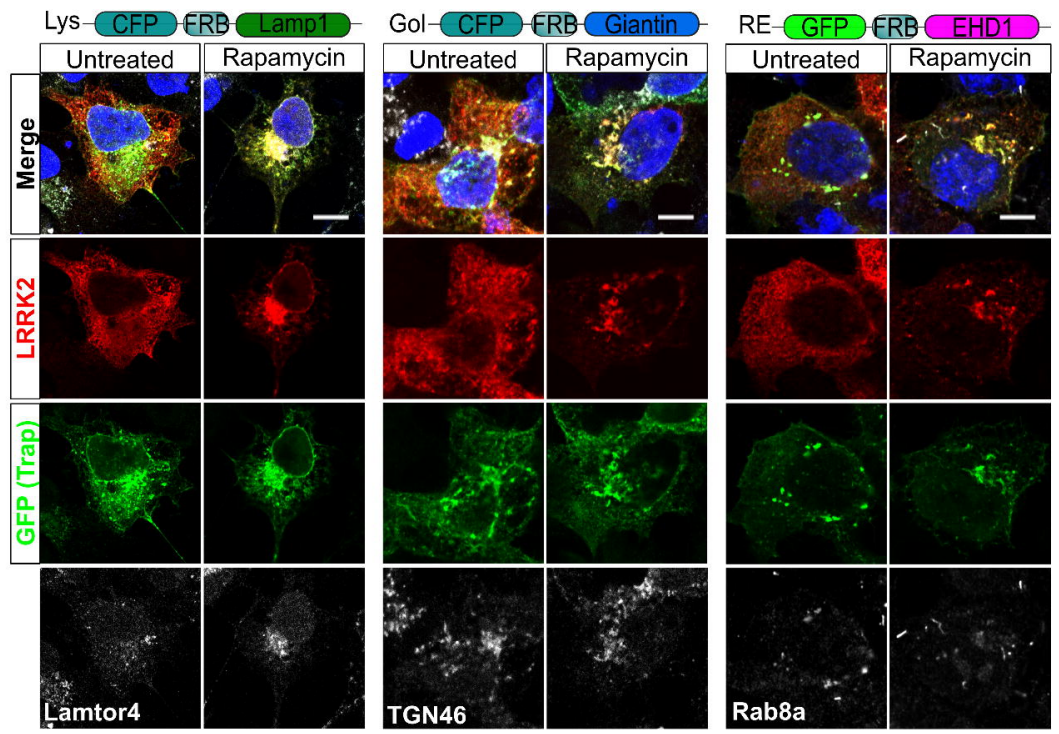

Figure 1 
A

Lys Gol

Untr. Rapa. Untr. Rapa. Untr. Rapa.
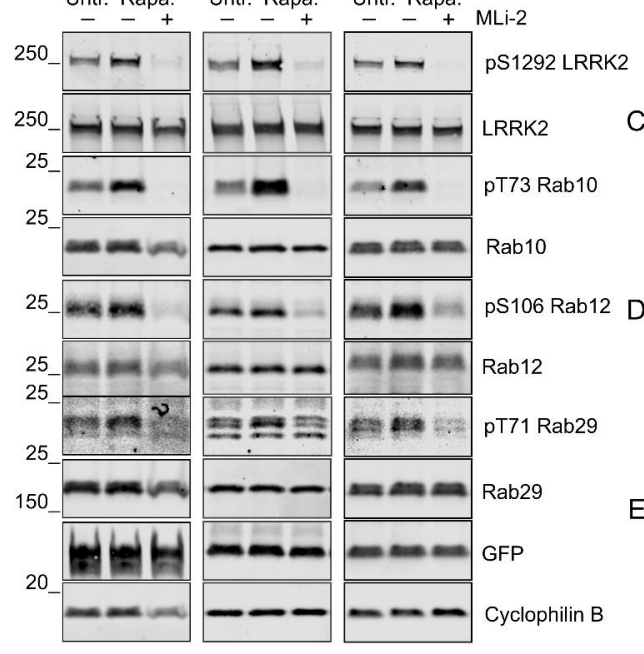

F

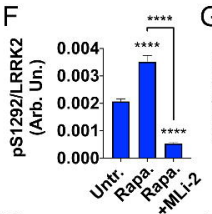

$\mathrm{H}$

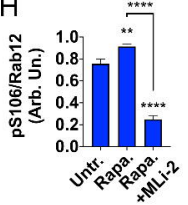

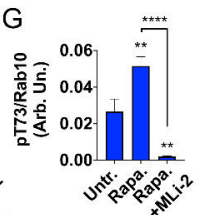

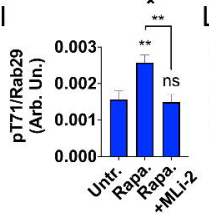

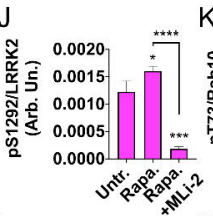
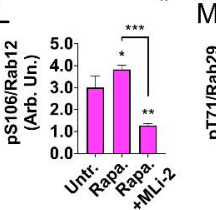
RE

B
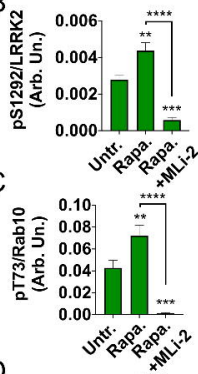

pS106 Rab12

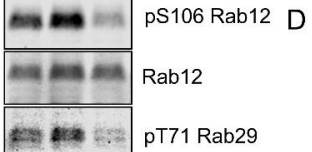

E

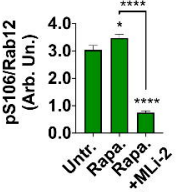

踣 0.004

单 0.00 占遂 0.001

Cyclophilin B

N
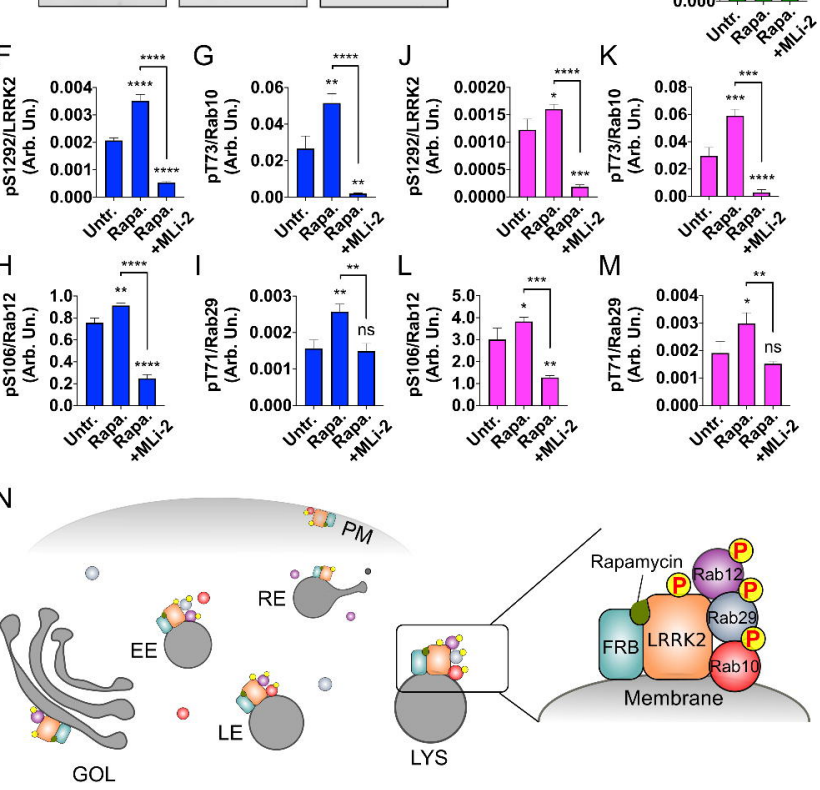

Figure 2

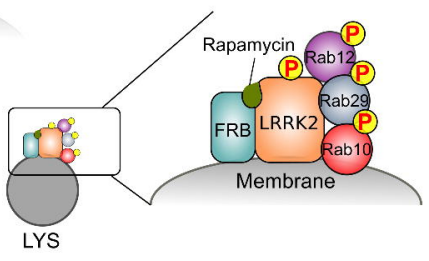



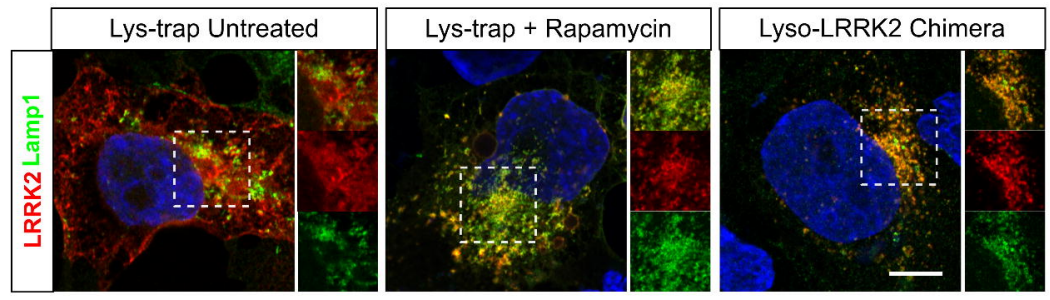

B

Untr. RapaUntr.
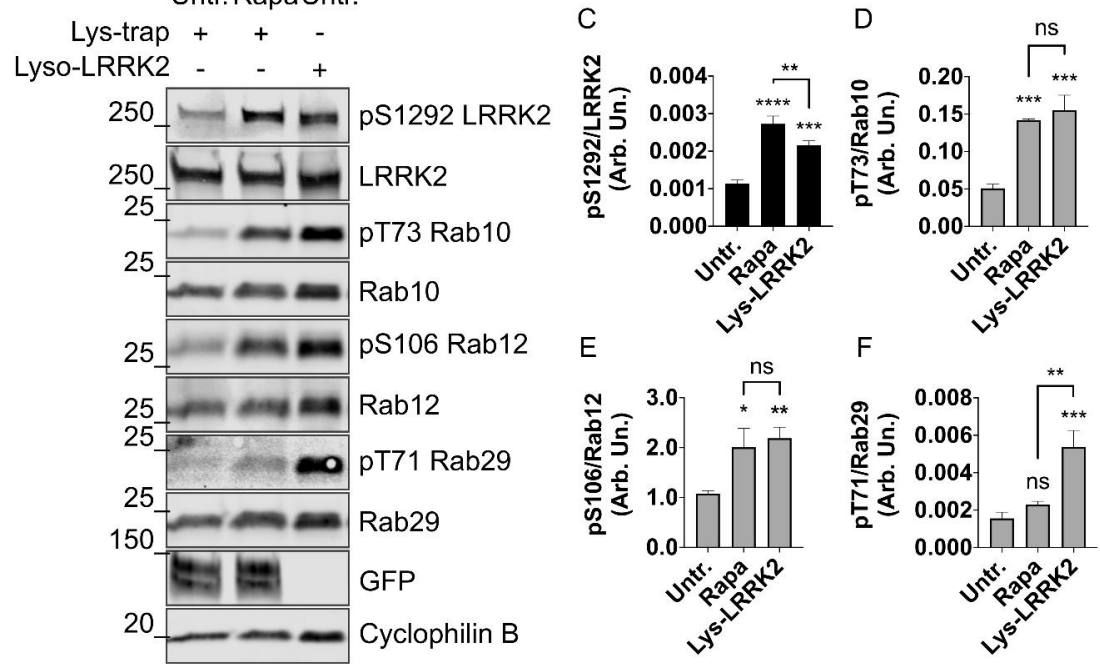

Figure 3 


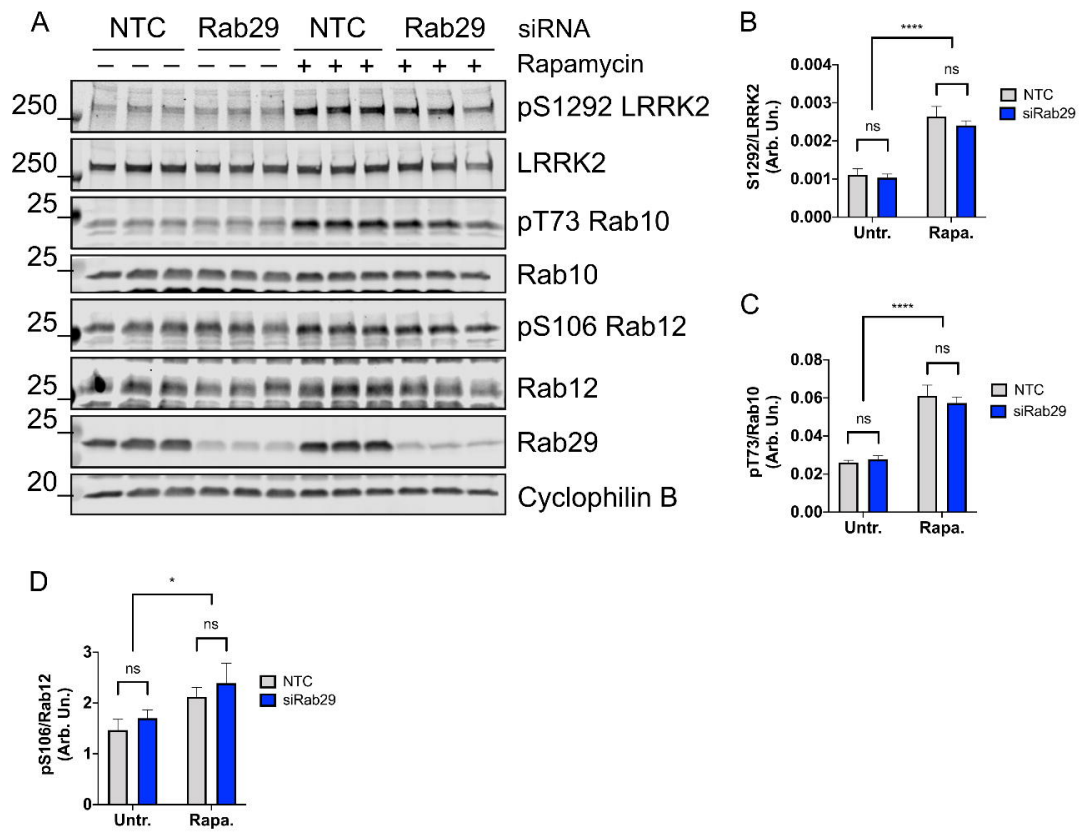

Figure 4 

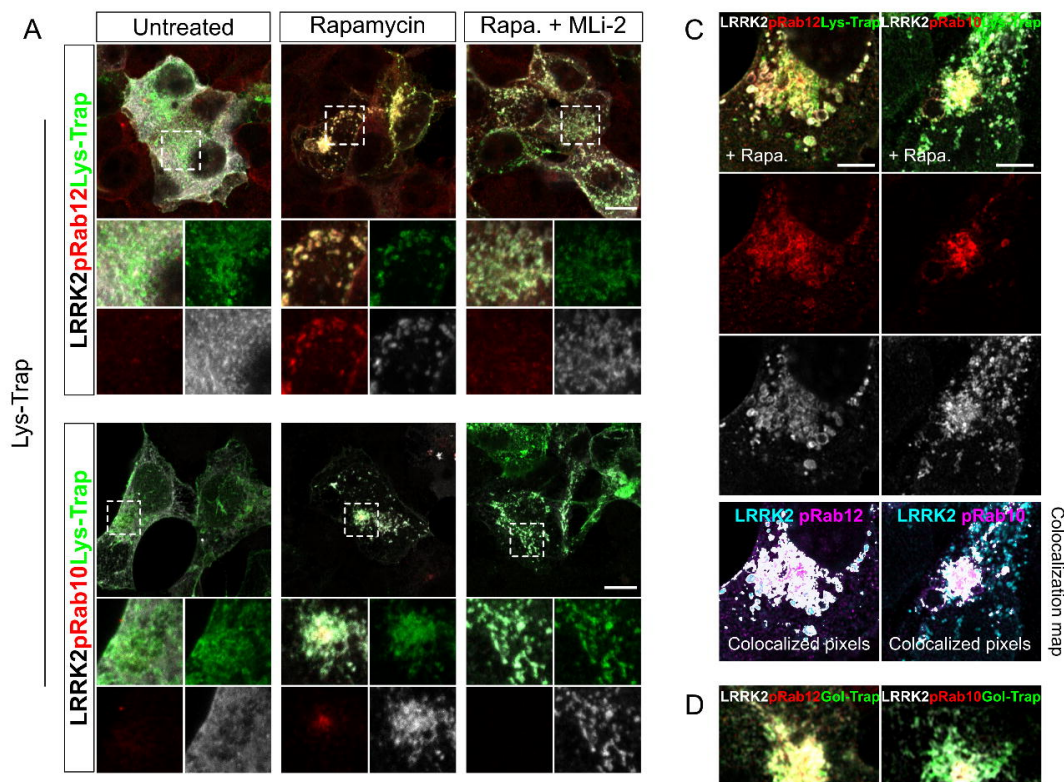

D

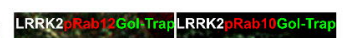

$B$
$B$
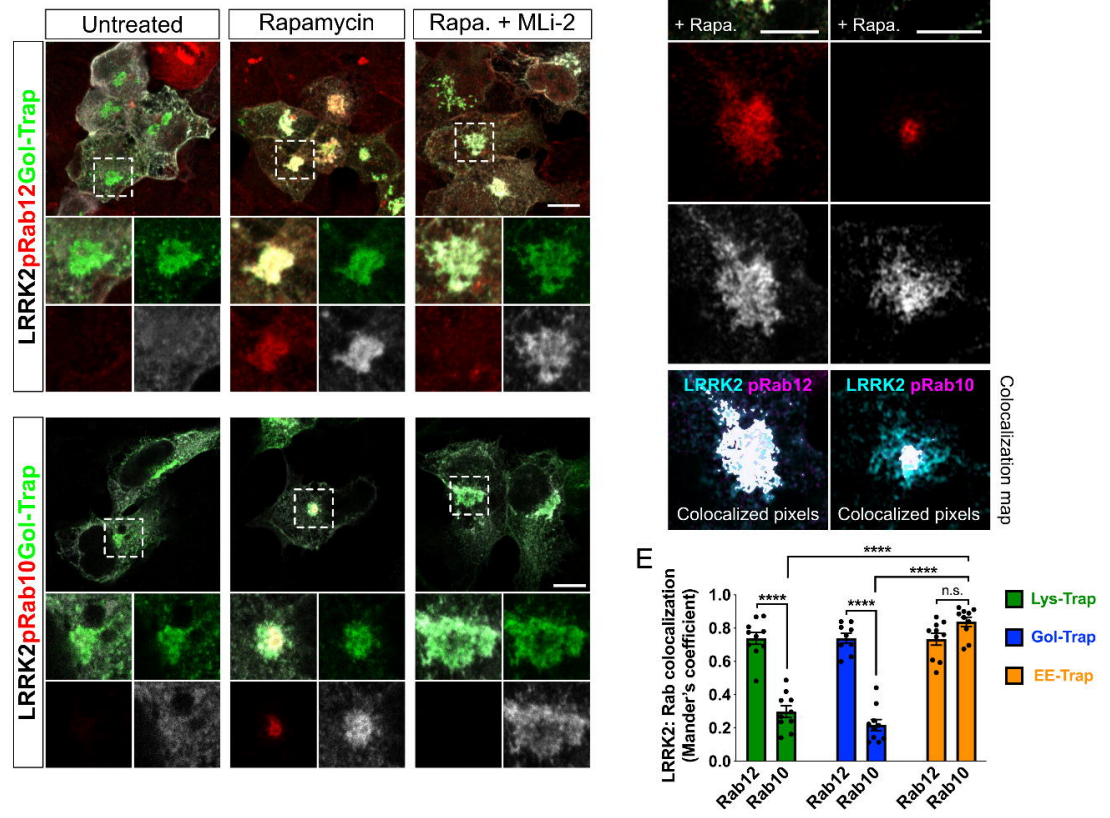

Figure 5 
A

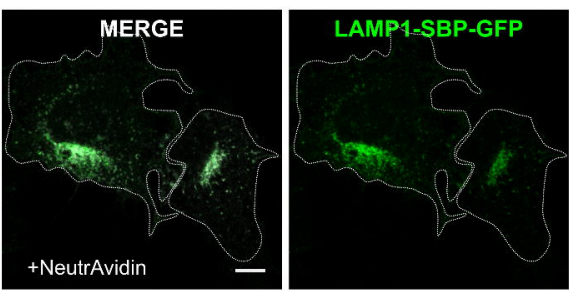

B
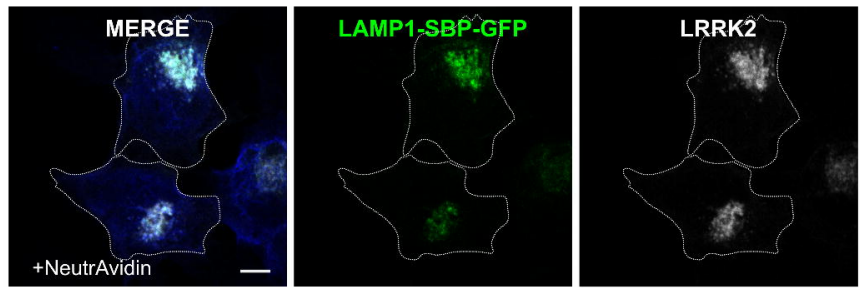

E

C

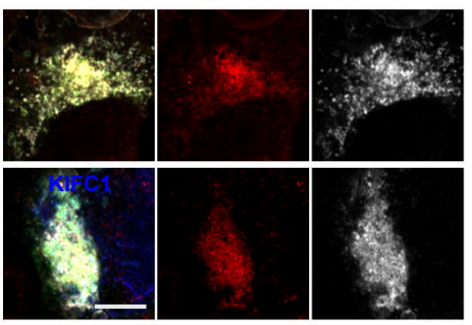

D

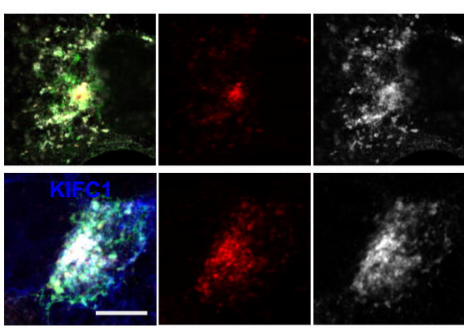

LRRK2

neser.
$F$
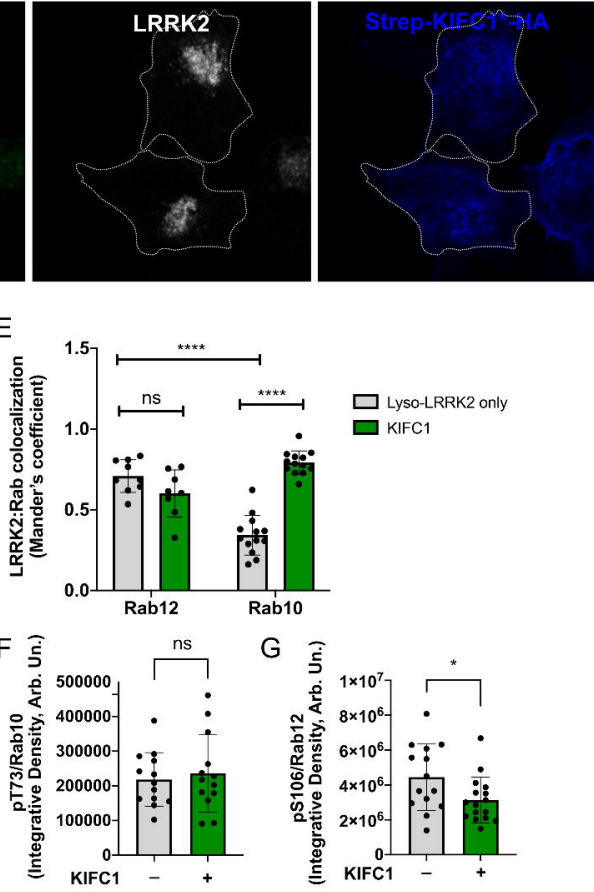

Figure 6 
A WT RC YC GS KM + + + + + + + + + + $--+--+--+--+--+$

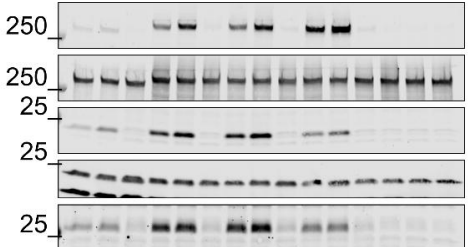
pS1292 LRRK2

LRRK2

pT73 Rab10

Rab10

pS106 Rab12

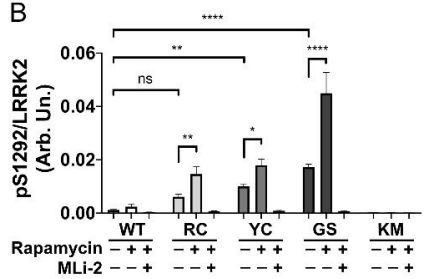

20

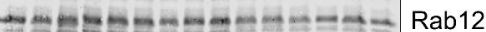

Cyclophilin B

C

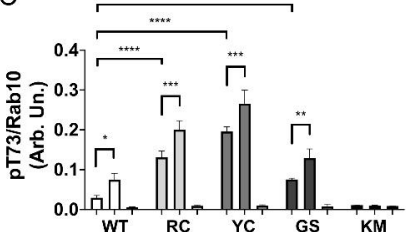

Rapamycin $\frac{\text { WT }}{-++} \frac{\mathrm{RC}}{-++} \frac{\text { YC }}{-++} \frac{\text { GS }}{-++} \frac{\mathrm{KM}}{-++}$ $\mathrm{MLi}-2--+--+--+--+--+$

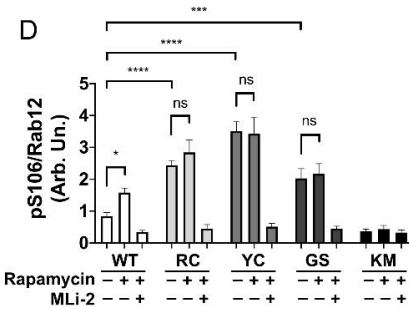

E $\quad$ WT $\frac{\text { RC }}{-++} \frac{\text { YC }}{-++++++} \frac{\text { GS }}{-++}$

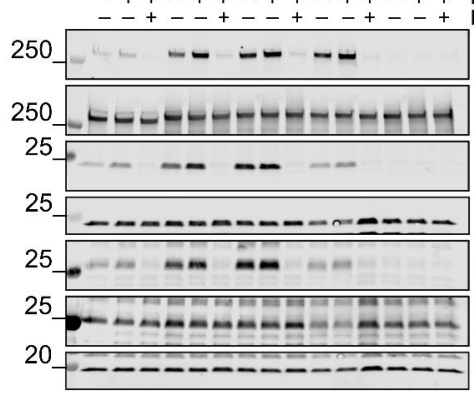

Rapamycin

MLi-2 pS1292 LRRK2

F

G

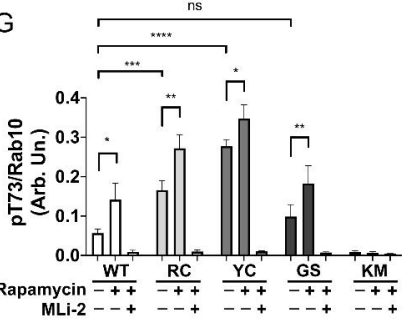

$\mathrm{H}$

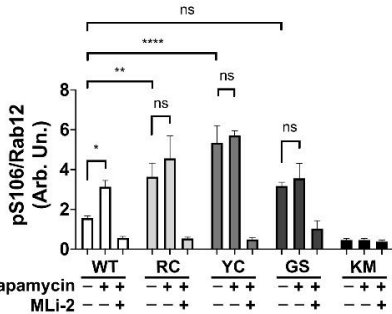

